\title{
Influenza Virus: A Master Tactician in Innate Immune Evasion and Novel Therapeutic Interventions
}

\author{
Alan Chen-Yu Hsu ${ }^{1,2 *}$ \\ ${ }^{1}$ Viruses, Infections/Immunity, Vaccines \& Asthma, Hunter Medical Research Institute, Newcastle, NSW, Australia, ${ }^{2}$ Priority \\ Research Centre for Healthy Lungs, Faculty of Health and Medicine, The University of Newcastle, Newcastle, NSW, Australia
}

\section{OPEN ACCESS}

Edited by:

Amy Rasley,

Lawrence Livermore National Laboratory (DOE), United States

Reviewed by: Katie Louise Flanagan, RMIT University, Australia Irving Coy Allen, Virginia Tech, United States

${ }^{*}$ Correspondence: Alan Chen-Yu Hsu alan.hsu@newcastle.edu.au

Specialty section: This article was submitted to Microbial Immunology, a section of the journal Frontiers in Immunology

Received: 09 January 2018

Accepted: 26 March 2018

Published: 12 April 2018

Citation: Hsu AC-Y (2018) Influenza Virus: A Master Tactician in Innate Immune Evasion and Novel Therapeutic Interventions.

Front. Immunol. 9:743. doi: 10.3389/fimmu.2018.00743
Influenza is a contagion that has plagued mankind for many decades, and continues to pose concerns every year, with millions of infections globally. The frequent mutations and recombination of the influenza A virus (IAV) cast a looming threat that antigenically novel strains/ subtypes will rise with unpredictable pathogenicity and fear of it evolving into a pandemic strain. There have been four major influenza pandemics, since the beginning of twentieth century, with the great 1918 pandemic being the most severe, killing more than 50 million people worldwide. The mechanisms of IAV infection, host immune responses, and how viruses evade from such defensive responses at the molecular and structural levels have been greatly investigated in the past 30 years. While this has advanced our understanding of virus-host interactions and human immunology, and has led to the development of several antiviral drugs, they have minimal impact on the clinical outcomes of infection. The heavy use of these drugs has also imposed selective pressure on IAV to evolve and develop resistance. Vaccination remains the cornerstone of public health efforts to protect against influenza; however, rapid mass-production of sufficient vaccines is unlikely to occur immediately after the beginning of a pandemic. This, therefore, requires novel therapeutic strategies against this continually emerging infectious virus with higher specificity and cross-reactivity against multiple strains/subtypes of IAVs. This review discusses essential virulence factors of IAVs that determine sustainable human-to-human transmission, the mechanisms of viral hijacking of host cells and subversion of host innate immune responses, and novel therapeutic interventions that demonstrate promising antiviral properties against IAV. This hopefully will promote discussions and investigations on novel avenues of prevention and treatment strategies of influenza, that are effective and cross-protective against multiple strains/subtypes of IAV, in preparation for the advent of future IAVs and pandemics.

Keywords: influenza, influenza A virus, virulence factors, hemagglutinin, polymerase acidic 1-F2, NS1, therapeutics

\section{INTRODUCTION}

Influenza A viruses (IAVs) and their continuous emergence/re-emergence are undoubtedly an important cause of global concern, morbidity and mortality, clinical, and socio-economical burden in humans. The emergence of highly pathogenic avian influenza virus H5N1 in 1997 and 2003, H7N7 in 2003, recent influenza H1N1 pandemic in 2009 (H1N1pdm09), and H7N9 in 2013 have caused tremendous mortality in the affected populations, and in our current inter-connected world, the concerns and impact of potential pandemics are truly global. 
The ongoing circulation of IAVs in their natural hosts and the ever-mutating antigenicity frequently leads to the appearance of novel viruses with the ability to cross species barriers and infect humans with unpredictable pathogenicity. Furthermore, effective immuno-evasive and modulatory strategies employed by IAVs have made it difficult for both the innate and acquired immunity to combat the infections without the help of vaccination.

The early innate immune responses to IAV infections are essential in the immediate control of viral replication and spread. However, IAVs have also evolved to produce only a handful, but multi-functional proteins to combat the intricate layers of human innate immune signaling pathways. These virulence factors inhibit host antiviral immunity, while stimulating inflammatory responses in the airways (cytokine storm) that cause severe symptoms. It is, therefore, imperative to understand the molecular mechanisms of IAV-mediated modulation of innate immunity by these viral factors in order to identify potential therapeutic options.

While antiviral drugs are mostly used either prophylactically or in treatment, they have minimal impact on the course of the disease. Heavy reliance on antiviral drugs has also placed a strong selective pressure on IAVs to mutate and develop resistance $(1,2)$. The unpredictable nature of novel IAVs and limited prevention and treatment options, therefore, urges the research and development of novel approaches against IAVs. This review summarizes the current understanding of the mechanisms of IAV infection in humans, the ways by which IAV suppresses antiviral immunity and causes the inflammatory cytokine storm, and novel peptidebased anti-influenza drugs that may potentially be beneficial as preventative and treatment strategies in current and future IAV pandemics.

\section{IAV-SIMPLE AND ELEGANT}

IAVs is a negative sense, single-stranded RNA virus $(\sim 80-120 \mathrm{~nm}$ in diameter) (3). The viral envelope features two surface glycoproteins, hemaglutinin (HA) and neuraminidase (NA), at a ratio of four HA to one NA (4). A small number of M2 ion channel is also embedded in the viral envelope at a ratio of one M2 channel to $10^{1}-10^{2}$ HA molecules (5) (Figure 1).

Within the envelope are the eight segments of influenza RNA, which are presented in a form of helical hairpin structure, each of which is coated with arginine-rich nucleoprotein (NP) (6-8). NP-RNA is complexed with heterotrimeric RNA-dependent RNA polymerase, which consists of three subunits, polymerase basic (PB)1, PB2, and polymerase acidic (PA) (3). Segment 1, 3, 4, 5, and 6 encodes for PB2, PA, HA, NP, and NA, respectively. Segment 2 encodes for $\mathrm{PB} 1$ and via a different reading frame an accessory protein PB1-F2 is expressed in most influenza A strains. Segment 7 encodes for matrix 1 (M1) protein and also M2 ion channel via alternative splicing. Segment 8 encodes for non-structural protein (NS)-1 and nuclear export protein. At
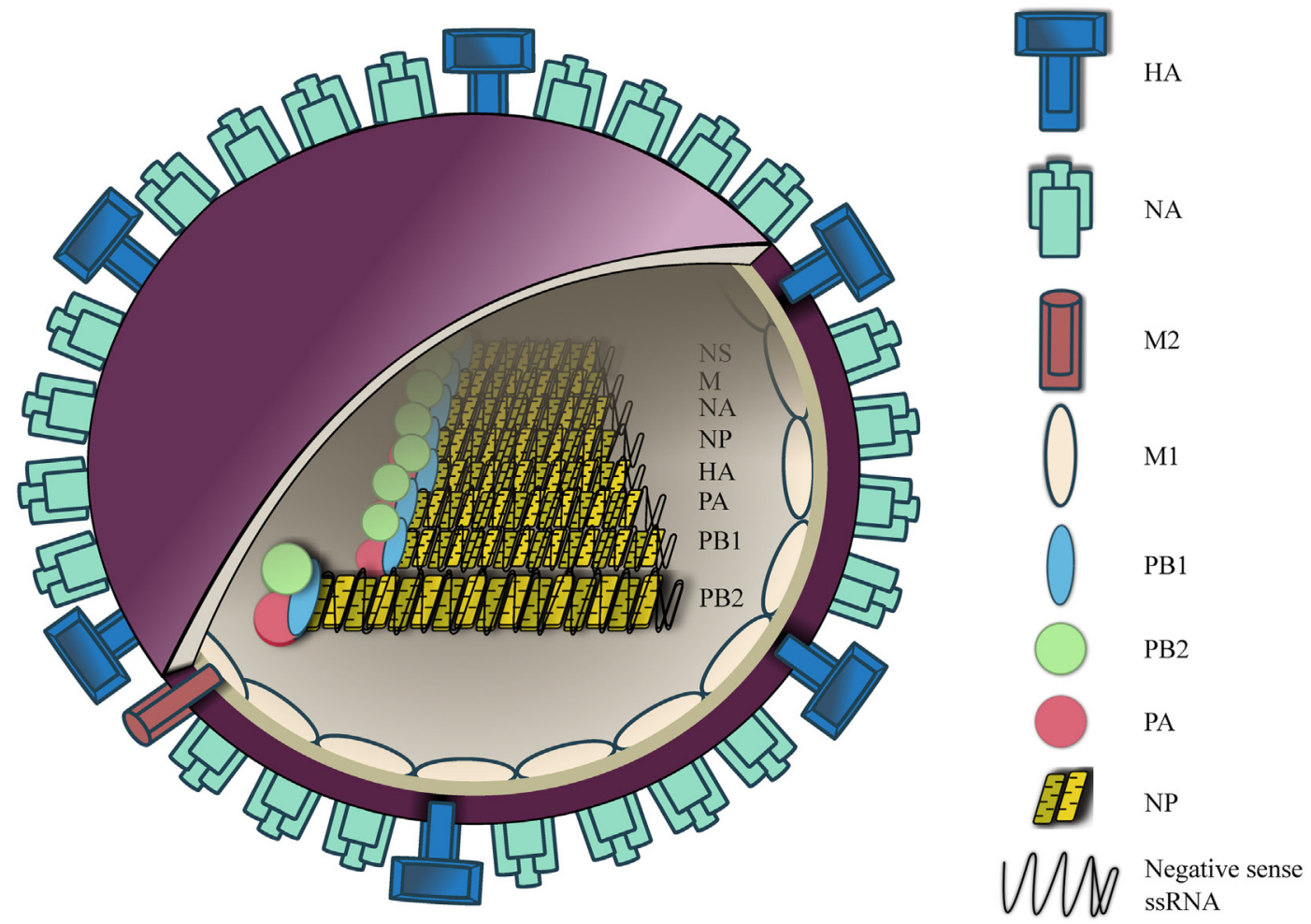

FIGURE 1 | Influenza A virus (IAV) structure. IAV contains an outer membrane envelope with hemaglutinin (HA), neuraminidase (NA), and matrix 2 (M2) ion channels, and an inner M1 protein layer that encloses an eight segmented RNA genome. The RNA segments are folded into a helical hairpin structure with nucleoprotein, which are complexed with heterotrimeric RNA-dependent RNA polymerase [polymerase basic (PB1), PB2, and polymerase acidic (PA)]. 
both $3^{\prime}$ and $5^{\prime}$ ends of each segment lies a non-coding region of varying length that acts as a promoter site for viral polymerase complex to initiate transcription. This region also contains an mRNA polyadenylation signal and a signal for virus assembly.

The primary site of infection by IAVs are epithelial cells of the respiratory mucosa. Airway epithelial cells are both susceptible and permissive to IAV infection. This occurs by the binding of HA to the sialyl sugar chain receptors on the host cell surface, allowing the virus to be internalized into endosomes in the host epithelial cells. The low $\mathrm{pH}$ environment of the endosome promotes HA to undergo conformational change that liberates and inserts a fusion peptide from the amino terminus of HA into the endosomal membrane. This spring-loaded mechanism fuses the viral envelope and the membrane together, thereby releasing viral RNP into the host cytoplasm $(9,10)$. The M2 ion channel also allows an influx of $\mathrm{H}^{+}$ions into the virion, and lowers the intra-virionic $\mathrm{pH}$. This in turn disrupts the viral RNP-M1 protein interaction and subsequently releases viral RNP into host cellular cytoplasm (11-14). Released IAV RNAs and polymerases are then translocated into the nucleus where viral replication occurs. The newly synthesized viral structural proteins and viral segments then traffic to lipid rafts on the plasma membrane to be released $(15,16)$. Since the viral envelope is derived from the host membrane, which contains sialic acid glycoproteins, the newly formed virion remains intact on the host cell surface. The viral NA cleaves the host cell surface sialic acid residues, releasing the newly formed virions free from the host cell surface.

\section{HA-STRUCTURALLY PLASTIC AND ABSOLUTELY ESSENTIAL FOR INFECTIVITY, HUMAN TRANSMISSION, AND PANDEMIC}

IAVs HA is responsible for the entry of the virus in the host cells by binding to host cell surface glycoproteins terminated with sialic acid residues at specific linkages. Human IAVs preferentially bind to glycoproteins containing the terminal SA $\alpha 2,6 \mathrm{Gal}$ linkage, which are predominately found in human upper airway epithelium (17-19). In contrast, avian IAVs bind to that with terminal $\mathrm{SA} \alpha 2,3 \mathrm{Gal}$ linkage in the lower airways (17-21).
This difference in binding specificity and distribution of sialic acid residues may in part explain why highly pathogenic avian influenza virus $\mathrm{H} 5 \mathrm{~N} 1$ is currently incapable of transmitting from human to human in a sustainable manner. In contrast, pig trachea contains both $\mathrm{SA} \alpha 2,6 \mathrm{Gal}$ and $\mathrm{SA} \alpha 2,3 \mathrm{Gal}$ linkages, indicating that pigs can act as an intermediate mixing host (22-24), allowing the reassortment of both avian and human viruses to occur. The prime example is the emergence of the $2009 \mathrm{H} 1 \mathrm{~N} 1$ pandemic, which was the result of triple reassortment between avian, swine, and human IAVs in pigs $(25,26)$. The difference in their binding specificity can be explained by the amino acid residue at position 226 of HA glycoprotein. HA of human influenza viruses contains a Leu226 that results in the preferential binding to $\mathrm{SA} \alpha 2,6 \mathrm{Gal}$ linkage. In contrast, $\mathrm{HA}$ of avian influenza viruses have a $\mathrm{Gln} 226$, which binds to SA $\alpha 2,3 \mathrm{Gal}$-linked glycoproteins $(27,28)$.

Two recent important investigations further characterized the molecular changes that are required for $\mathrm{H} 5 \mathrm{~N} 1$ to become transmissible among humans. Imai et al. showed that Gln226Leu/Gly228Ser increased the binding of H5 HA to $\mathrm{SA} \alpha 2,6 \mathrm{Gal}$ while retaining the binding capacity to $\mathrm{SA} \alpha 2,3 \mathrm{Gal}$. When Asn158Asp/Asn224Lys/Thr318Ile was introduced with Gln226Leu/Gly228Ser, this fully converted the virus with H5 HA in an H1N1pdm09 backbone to achieve sustainable aerosol transmission in a ferret model (29). In addition, Herfst et al. also demonstrated that mutant H5N1 containing His103Tyr, Thr156Ala, and Gln222Leu in the HA protein, and Glu627Lys in the viral polymerase protein $\mathrm{PB} 2$ was able to efficiently transmit between ferret models via aerosols (30). Interestingly, although not surprising, most of these residues (Asn158Asp; Gln222Leu; Asn224Lys; Gln226Leu; Gly228Ser) are at the sialic acid residue binding site of the globular domain.

When these amino acid residues in H5N1 HA were changed in silico to the ones in the airborne-capable HA, and structurally modelled using the wild-type H5N1 HA as a template in SWISSMODEL (31), the binding cavity in the mutant, surrounded by the 130-, 190-, and 220-loop, may potentially be lengthened and result in a slightly larger binding cavity (Figure 2). Furthermore, Asn158Asp is also likely to make the receptor binding pocket slightly more acidic, further facilitating a more efficient airborne transmission. A mutant $\mathrm{H} 1 \mathrm{~N} 1$ with Tyr17His ( $\mathrm{pH}$ 6.0) in HA
A A/Auckland/1/2009 (H1N1)

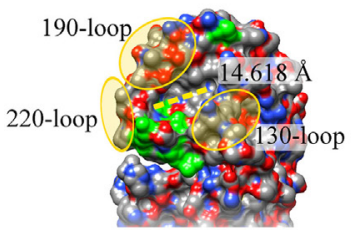

B A/Vietnam/1203/2004 (H5N1)

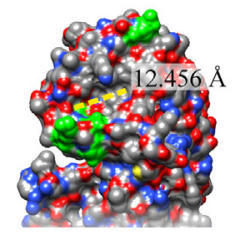

C

A/Vietnam/1203/2004 (H5N1) - N158D/N224K/Q226L/G228S

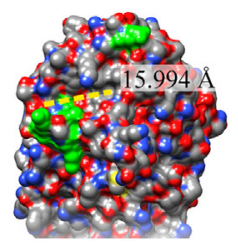

FIGURE 2 | Crystal structure of hemaglutinin (HA) sialic acid residue binding site of H1N1pdm09, H5N1, and airborne-transmissible H5N1. The sialic acid residue binding site on the HA of (A) AWashington/5/2011 (H1N1pdm09; PDB: 4LKX), (B) ANietnam/1203/2004 (H5N1; PDB: 2FKO), and (C) H5N1 with Asn148Asp, Gln222Leu Asn224Lys Gln226Leu, and Gly228Ser. These amino acid residues were changed in silico and modeled using HA of H5N1(PDB: 2FK0) as a template in SWISS-MODEL. Protein visualization and distance calculation was performed in UCSF Chimera molecular visualization application. 
showed loss of airborne transmission, and was less efficient in contact transmission with reduced pathogenicity in mice, and His17Tyr ( $\mathrm{pH}$ 5.3) revertant virus regained the airborne transmissibility and pathogenicity (32). This may also widen the binding capacity toward moieties with other linkages/modifications. While changes in these residues are important in airborne transmission in ferret models, it remains unclear whether similar or other changes in HA need to occur for a sustainable airborne transmission in humans, these studies provide valuable insight into cause of efficient human transmission, surveillance for potential human transmissible IAVs, and new approach into therapeutic designs.

Epidermal growth factor receptor, a glycoprotein with potential terminal SA $\alpha 2,6 \mathrm{Gal}$ and SA $\alpha 2,3 \mathrm{Gal}$ linkages $(33,34)$, has been shown to be important in IAV entry into host cells in a ligand (EGF)-independent, and phosphoinositide-3 kinase (PI3K)-activation-dependent manner (35-37). However, IAV has also been demonstrated to cause infection in the absence of its respective receptors. $\mathrm{H} 3 \mathrm{~N} 2$ and $\mathrm{H} 1 \mathrm{~N} 1$ was able to infect and replicate to the similar titer in the lung of the mice lacking receptors with $\mathrm{SA} \alpha 2,6 \mathrm{Gal}$ linkages compared to wild type mice (38). Consistent with this finding, while human bronchial epithelial cells showed higher levels of $\mathrm{SA} \alpha 2,6 \mathrm{Gal}$ compared with $\mathrm{SA} \alpha 2,3 \mathrm{Gal}$ linkages, human IAV H3N2, and a low pathogenic avian H11N9 have been shown to enter into bronchial epithelial cells at a similar rate (39). This indicates that sialic acid residues may not be absolutely essential in influenza virus entry, and other moieties, such as sulfonated or fucosylated glycoproteins may also be targeted by HA protein $(40,41)$.

\section{THE POLYMERASE CONSTELLATION}

IAV RNA is always packaged in a heterotrimeric RNA-dependent RNA polymerase complex with viral polymerase basic (PB)1, $\mathrm{PB} 2$, polymerase acidic (PA), and nucleoprotein (NP), and upon successful entry into epithelial cells, this complex translocates to the nucleus where replication occurs. As IAV viral RNA does not contain a $5^{\prime}$ cap as they do on host RNAs, PB2 contains a capbinding domain that binds to the $5^{\prime}$ cap of nascent host RNAs, and PA then "steals" the cap off the host RNA by cleavage, which is then used as primers to initiate transcription of viral mRNAs. This process is called cap-snatching. This polymerase complex, therefore, is critical in the survival of IAVs, and has been implicated in the host range determination. Clements et al. demonstrated that the reassortment virus containing the PB2 gene of avian origin and all other genes from human IAV is able to replicate efficiently in the avian host, while showing restricted replication in the mammalian respiratory tract (42). This reassortment virus was then progressively passaged to generate mutant viruses that were able to replicate in mammalian tissues. Nucleotide sequence analysis revealed that this phenotype of the reassortment virus was due to the Glu627 of the PB2 gene (43). A single amino acid substitution to a lysine residue allowed this mutant reassortment virus to replicate efficiently with increased pathogenicity in the mammalian system (44-47). All the avian IAVs analyzed to date have a Glu627 in the PB2, whereas human IAVs have a Lys627, indicating this residue at position 627 of PB2 is an important host range determinant of IAVs (43). Furthermore, Glu627Lys substitution being a prerequisite for aerosol to airborne transmissibility switch is troubling, as the viruses isolated from the recent fatal cases of H7N9 infection in China carried Glu627Lys substitution, but the viruses isolated from poultry did not $(48,49)$.

The compatibility of other subunits of the polymerase complex is also involved in the host range specificity $(50,51)$. Reassortment viruses with human PA and avian PB1 and PB2, or with human $\mathrm{PA}$ and $\mathrm{PB} 2$ and avian $\mathrm{PB} 1$ showed a restricted viral replication in mammalian cells, indicating that a specific constellation of polymerase genes may be involved in the host range specificities (51).

\section{PB1-F2-A PYROGENIC DEATH DEALER}

In the middle of searching for an IAV-derived out-of-frame polypeptide that can be recognized by $\mathrm{CD} 8^{+} \mathrm{T}$ lymphocytes, an 11 th gene, PB1-F2, was serendipitously discovered (52). PB1-F2 is an accessory protein that is translated from an alternative +1 open reading frame of $\mathrm{PB} 1$. It modulates innate immune responses at multiple signaling levels, leading to increased inflammatory and impaired antiviral responses. PB1-F2 contains a mitochondrial targeting sequence at the carboxyl-terminus and has a proapoptotic effect on immune cells (53). By binding to the inner and outer mitochondrial membrane transport protein adenine nucleotide translocator 3 and voltage-dependent anion channel 1 (VDAC1), PB1-F2 disrupts mitochondrial integrity, releasing cytochrome $\mathrm{C}$, and leading to apoptosis. Another study also demonstrated that PB1-F2 can be translocated into the inner mitochondrial space in a TOM40-dependent manner, and impair mitochondrial function (54).

Host innate immune response is triggered by the pattern recognition receptors including retinoic acid inducible gene 1 (RIG-I). RIG-I binds to IAV RNAs and forms a complex with the adaptor protein tripartite motif-containing protein (TRIM)25 (55). This complex interacts with mitochondrial antiviral signaling (MAVS) protein to induce the activation of interferon (IFN)-regulatory factor (IRF) 3, which initiates the induction of important antiviral cytokines including type I and III IFNs $(56,57)$. These IFNs then stimulate the expression of over 300 IFN-stimulated genes (ISGs) such as protein kinase $\mathrm{R}$ (PKR) to limit viral replication (58). Toll-like receptor 3 also recognizes IAV RNAs and activates an important transcription factor nuclear-factor-kappa-B (NF- $\kappa \mathrm{B})$, which stimulates the expression of predominantly inflammatory cytokines, including interleukin (IL)-6 and IL-1 $\beta$ (59-61).

PB1-F2 has also been shown to inhibit RIG-I-TRIM25mediated antiviral signaling pathway by direct interaction with MAVS $(62,63)$. Furthermore, this inhibition of type I and III IFNs was enhanced with Asn66Ser substitution. Intriguingly, PB1-F2 can also be expressed as a truncated ( 57 amino acids) protein devoid of the carboxyl terminus, which failed to translocate into the mitochondria (54). The full length PB1-F2 is mostly expressed by highly pathogenic viruses, such as H5N1, whereas low pathogenic subtypes, such as H1N1 (except PB1-F2 of 1918 $\mathrm{H} 1 \mathrm{~N} 1$ ) tend to express this truncated form. PB1-F2 also enhances the development of secondary bacterial pneumonia in mice with reduced survival rate. When $\mathrm{PB} 1-\mathrm{F} 2$ of $\mathrm{A} / \mathrm{PR} / 8$ was engineered to 
become that of $1918 \mathrm{H} 1 \mathrm{~N} 1$ by mutagenesis, the resulting mutant virus became more pathogenic with and without secondary bacterial infection (64). Surprisingly, this increased pathogenicity by PB1-F2 of $1918 \mathrm{H1N} 1$ was also attributed to Asn66Ser (65). A mutant virus carrying H5N1 PB1-F2 gene with Asn66Ser in A/WSN/33 background led to increased pathogenicity with 100fold increase in viral replication compared with wild-type virus in mice. Similarly, when the $1918 \mathrm{H} 1 \mathrm{~N} 1$ virus was reconstructed to carry a pathogenicity-reducing substitution (Ser66Asn), the resulting virus led to attenuated viral replication and reduced mortality.

PB1-F2-mediated IAV pathogenicity could be the result of its interaction with NLRP3-inflammasome. PB1-F2 of H7N9 has been shown to form fibrillar higher molecular weight aggregates that were incorporated into the phagolysosome and induced ASC speck formation upon acidification (66). This resulted in the activation of NLRP3-mediated inflammasome, leading to increased production of pyrogenic cytokine IL-1 $\beta$ and enhance the pathogenesis of influenza viral pneumonia in mouse models $(66,67)$. PB1-F2 of avian H7N9 has also been shown to increase mitochondrial reactive oxygen species (ROS) and calcium $\left(\mathrm{Ca}^{2+}\right)$ efflux, which contributed to the activation of NLRP3 inflammasome (68).

PB1-F2, therefore, appears to be a major driving force for excessive pro-inflammatory cytokine storm and pathogenesis (Figure 3), and Asn66 is important in the function of PB1-F2. Nevertheless, how IAV-mediated inflammatory cytokine storm holds an advantage in IAV survival remains elusive. The formation of this fibrillary PB1-F2 aggregate is consistent with the crystal structural data that indicated a strong propensity for PB1-F2 to adopt an oligomerization structure and form membrane pores in planar lipid bilayers $(69,70)$. However, it remains unknown whether it is a requirement for PB1-F2 to form this fibrillar structure to interact with all of its binding targets, and while the oligomerization domain is located in carboxyl-terminal $\alpha$-helix, it is unknown if oligomerization is dependent on Asn66. It is also unclear if this structure is unique to highly pathogenic strains or is present in all subtypes/strains.

\section{NS1 - THE INVERSE INTERFERON}

When IFNs were first described by Isaacs and Lindenmann, it was found that cells treated with heat-inactivated, but not live IAV could produce these antiviral cytokines, an immuno-inhibitory phenomenon he described as "inverse interference" $(71,72)$. The identity of Lindenmann's inverse interferon was later revealed to be NS1 protein. NS1 protein is encoded by the NS gene together with nuclear export protein via alternative splicing. As NS1 is encoded by the shortest gene of all, NS1 protein is rapidly expressed to high levels in the infected epithelial cells $(73,74)$.

NS1 is a multi-functional protein with a RNA-binding domain at its amino-terminus (residue 1-73), and an effector domain (residue 74-230) at the carboxyl-terminus $(73,75,76)$. The RNA-binding domain of NS1 recognizes dsRNA sequences and blocks the host RNA detection system. The effector domain of NS1 can stabilize the RNA-binding domain, but it predominantly interacts with host cellular proteins and interferes with host mRNA processing and host innate immune responses (Figure 4). NS1 inhibits host pre-mRNA endonucleolytic cleavage and polyadenylation by direct interaction and inhibition of cleavage and

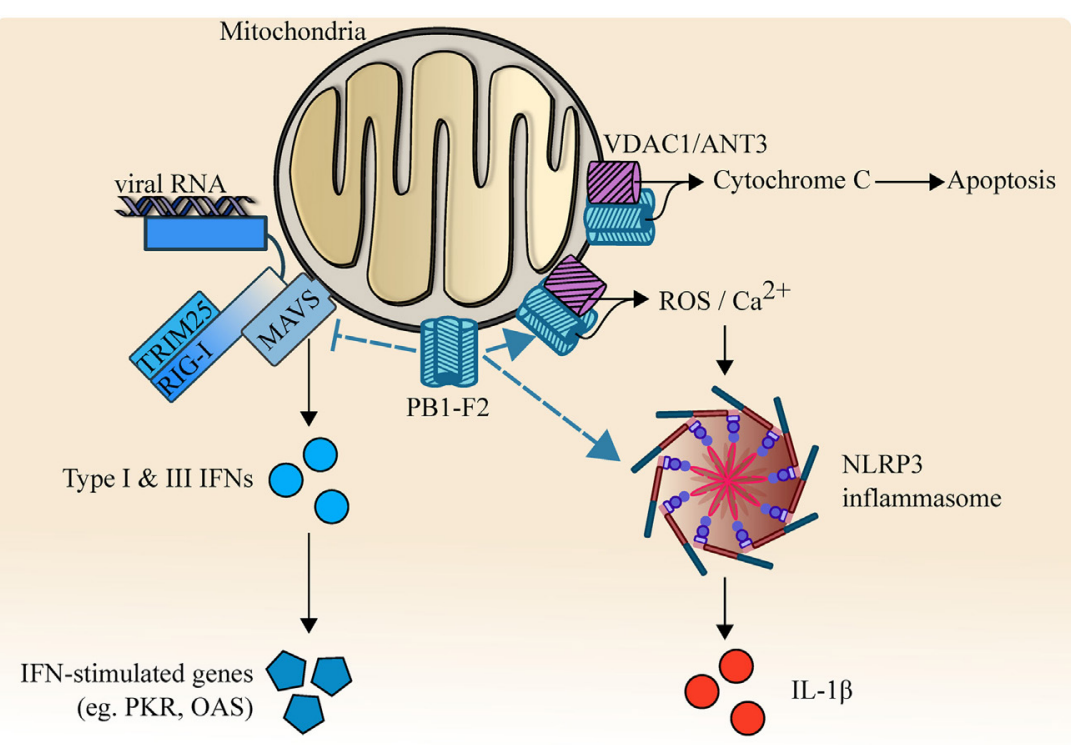

FIGURE 3 | PB1-F2 and its multi-functional functions. PB1-F2 binds and inhibits RIG-I/TRIM25/MAVS-mediated signaling activity, reduces the transcription of type I, III interferon (IFNs), and the downstream induction of antiviral responses. PB1-F2 also forms fibrillar higher molecular weight aggregates that facilitate the release of pyrogenic IL-1 $\beta$ by activating NLRP3-dependent inflammasome. Outer mitochondrial membrane ion channel voltage-dependent anion channel 1 (VDAC1) and adenine nucleotide translocator 3 (ANT3) are also targeted by PB1-F2, leading to increased release of cytochrome C, reactive oxygen species (ROS), and Ca ${ }^{2+}$. These result in apoptosis and NLRP3-inflammasome activation. 


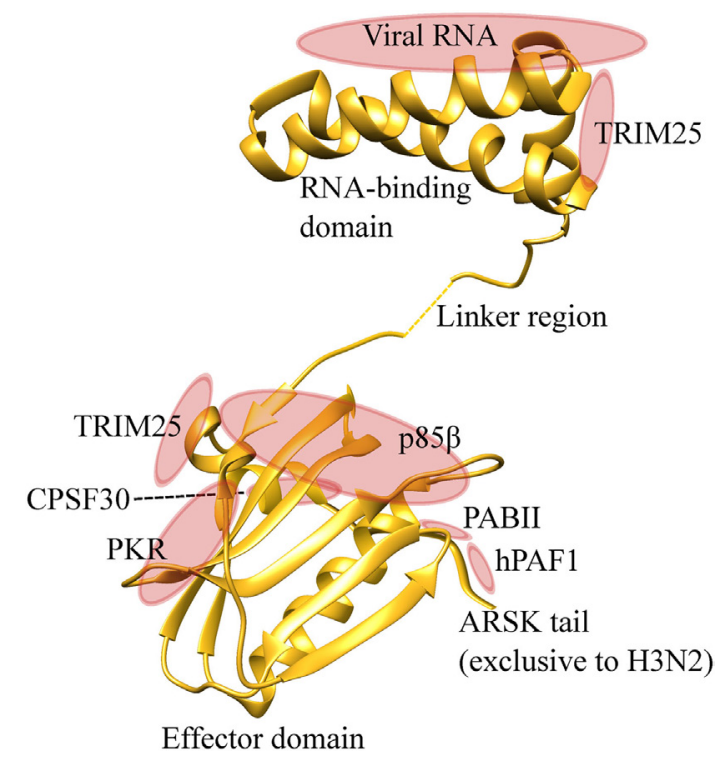

FIGURE 4 | NS1 is an effective suppressor of host antiviral immunity. NS1 protein contains an amino-terminal RNA binding domain and carboxylterminal effector domain. RNA binding domain binds to viral RNAs and prevents detection by the host pattern recognition system. The effector domain binds to TRIM25, and inhibits RIG-I-MAVS interaction and downstream induction of type I and III IFNs. This domain also interacts with protein kinase $\mathrm{R}(\mathrm{PKR})$, therefore, inhibiting its pro-apoptotic activities. $\mathrm{PI} 3 \mathrm{~K}-\mathrm{p} 85 \mathrm{\beta}$ subunit is also targeted by the effector domain to inhibit apoptosis. NS1 binds with CPSF30 and poly A binding protein II (PABII), inhibiting host protein synthesis and facilitating viral protein synthesis. The ARSK tail is exclusively found in H3N2, and directly binds with hPAF1 complex and inhibits the transcription of antiviral genes. Protein visualization was performed in UCSF chimera molecular visualization application.

polyadenylation specificity factor -30 subunit (CPSF30) and poly A binding protein II (77-79). By shutting down the host cellular protein synthesis in the infected cells, this helps the virus to gain control of the host machineries required for efficient viral protein synthesis.

The major function of NS1 is to antagonize host innate immune responses during infection, and this occurs at multiple stages of the IFN signalling cascade. In the nucleus, NS1 of human IAV H3N2 was recently shown to contain carboxyl-terminal ARSK tail sequence (amino acids 226-229), which is analogous to the ARTK sequence on the lysine 4 of histone H3 (H3K4) (80). This ARSK tail could act as a H3K4 histone mimic that directly interacts with human polymerase II-associated factor 1 complex and impairs the transcription of antiviral genes. However, this ARSK tail appears to be unique to $\mathrm{H} 3 \mathrm{~N} 2$, and is absent in H5N1, H7N9, and H1N1pdm09. In the cytoplasmic space, NS1 inhibits RIG-Imediated signaling and subsequent induction of type I IFNs (81, 82). Specifically, the NS1 protein inhibits RIG-I ubiquitination mediated by TRIM25, which is crucial for maximal type I and III IFNs expression during viral infection (83). This NS1-TRIM25 binding event is dependent on the Arg38 and Lys41 in the RNA binding domain, and Glu96 and Glu97 in the effector domain of NS1 $(84,85)$. NS1 protein also interferes with functions of important intracellular antiviral ISGs, including PKR and oligoadenylate synthase (OAS). The RNA-binding domain of NS1 can bind to viral RNA and avoid detection by PKR (86). It also binds to PKR itself via the effector domain (residue 123-127) and inhibits PKR-mediated viral mRNA suppression and apoptosis (87-90). OAS, which detects and cleaves viral RNA by activating RNase L, can also be blocked by influenza NS1. The RNA-binding domain of NS1 can out-compete the RNA binding capacity of OAS, thereby inhibiting the antiviral response (91). Another important target of NS1 is the PI3K signaling pathway. NS1 activates PI3K pathway by direct interaction with $\mathrm{p} 85 \beta$ subunit, thereby increasing the rate of viral internalization and inhibition of apoptosis. NS1-p85 $\beta$ interaction is dependent on Tyr89/Met93 (92), Leu141/Glu142 (93), and Pro164/Pro167 (94) of the effector domain, all of which are located adjacent to each other within a cleft between the two NS1 monomers. The anti-apoptotic effect of NS1-PKR and NS1-p85 $\beta$ interaction, however, appears to conflict with the pro-apoptotic function of PB1-F2. The molecular equipoise of pro- and anti-apoptotic response by these two contradicting signals and the timing of apoptosis remain elusive.

The carboxyl-terminus of the effector domain is essential in the NS1-mediated inhibition of antiviral responses. A mutant H7N7 virus that lacked NS1 gene or with a large carboxylterminus deletion showed impaired IFN suppression activity and attenuated replication in mammalian and avian epithelial cells (95). Similar findings were also observed with influenza viruses of different species. Both swine and turkey influenza virus with NS1 carboxyl-terminal truncation showed attenuated replication and an increase in IFN response compared to the wild type infection in its respective epithelial cells $(96,97)$. When the NS1 gene of high pathogenic avian $\mathrm{H} 5 \mathrm{~N} 1$ was replaced with the one that had a natural deletion of residues 191-195 from low pathogenic swine $\mathrm{H} 5 \mathrm{~N} 1$, the resulting virus was attenuated in viral replication and its IFNs inhibition in chickens (98). The deleted residues were then engineered into this NS1 and the resulting virus gained virulence, demonstrating the importance of these residues at 191-195 in IFN inhibition. The overall suppression of host antiviral immunity by the NS1 protein appears to be more effective with human IAV H3N2 compared with a low pathogenic subtype (39). This difference in the levels of inhibition by the NS1 proteins was likely due to differences in the amino acid residues discussed previously, which might be driven by the selective pressure and the long evolution of the virus in human populations. The H5N1 NS1 was able to dramatically reduce the induction of antiviral cytokines, leading to much higher viral replication $(99,100)$. Influenza NS1 is no doubt a powerful and fast-deployable antagonist that rapidly controls critical host immune infrastructure (Figures 3 and 4). The dual wields of NS1 and PB1-F2 thus allow for maximal viral replication with minimal interference from the host immune system, while causing severe disease in the infected individuals.

\section{NOVEL THERAPEUTIC STRATEGIES}

Influenza vaccination remains the cornerstone of efforts to protect against influenza, however, there are serious concerns regarding current vaccine prediction and manufacturing process. Mis-match or unforeseeable mutations between the circulating strain and the 
vaccine strain often results in compromised herd immunity and increased infections in that year (101). Vaccine manufacturing is a slow and problematic process, and this issue became evident during the $2009 \mathrm{H} 1 \mathrm{~N} 1$ pandemic. The first vaccines only became available 5 months after the identification of the virus, and mutations can occur during the vaccine manufacturing period, leading to reduced effectiveness than anticipated. Antiviral drugs, such as M2 channel and neuraminidase inhibitors, are mostly used as treatment for influenza. However, these drugs were mostly given to patients who had symptoms after the virus had shed, therefore, had limited effect. Neuraminidase inhibitors have been shown to be more effective in reducing length of hospital stay than a reduction of mortality rate $(102,103)$. Heavy use of these drugs has also resulted in a massive development of resistance, including the emergence of oseltamivir-resistant H1N1pdm09 (A/Newcastle/1/2011) in Newcastle, NSW, Australia (2). There is, therefore, an urgent need to identify and develop novel therapeutic targets for influenza, in preparation for future emergence of influenza viruses and pandemics.

The peptide-based therapeutics is an ideal approach that offers specificity with relatively minor side-effects, and can be administered directly to the lung via the pulmonary delivery route. This delivery route also increases the adsorption area in the lung by the therapeutic peptides. For these reasons, a number of peptides have been discovered to inhibit IAV replication at different stages of infection.

IAV HA represents an initial and critical step to a successful infection, and would be an ideal target for drug design as a preventative strategy. By screening a HA fragment peptide library, Chen and Guo identified a HA-targeting peptide, HA-pep25, that inhibited influenza virus binding and infection, including human H1N1, human and avian H5N1, and avian H7N9 (104). HA-pep25 is an 18-mer mimicking peptide that corresponds to the sialic acid residue binding site $\left(\mathrm{HA}_{221-238}\right)$, and specifically binds to the sialic acid residues, thereby shielding the cells from the viral attachment. Jones et al. also demonstrated that a 20-amino acid peptide derived from fibroblast growth factor 4 was able to inhibit viral binding to sialic acid residues by several IAVs, including H1N1, H3N2, and H5N1 (105). Prophylactic treatment in mice substantially reduced viral replication, and post-infection treatment was also as effective as rimantadine in protection against $\mathrm{H} 5 \mathrm{~N} 1$ in mice. Other peptide-based inhibitors, such as Flupep and Flufirvitide, have also been shown to be effective in inhibiting viral attachment to the cells. Flupep interacts with HA and inhibits viral binding (H1N1pdm09, H3N2, and H5N1) to the cell membrane (106). Flufirvitide is a mixture of hydrophobic $\alpha$-helical peptides that also binds with HA and blocks viral internalization and infection, and is currently being tested in clinical trials (107).

A class of synthetic anti-lipopolysaccharide peptides (SALPs) has been reported to also inhibit IAV attachment to cell surface. SALPs were originally designed to inhibit bacteria-mediated lethal septic shock in mice (108), but also possess antiviral activities against IAVs. Specifically, SALP PEP19-2.5 peptide showed high binding affinity toward the sialic acid residues, and led to reduced viral attachment and internalization, including human H3N2, H1N1pdm09, and high pathogenic H7N7 (109). Given that primary IAV infection is frequently followed by secondary bacterial infection, this class of peptides would be an ideal therapeutic approach for IAVs as a preventative or post-infection treatment. IAV polymerase complex is also an ideal therapeutic target due to its importance in viral protein synthesis in the host cells. $\mathrm{PB} 1_{1-25}$ and $\mathrm{PB} 1_{715-740}(110)$, and $\mathrm{PB} 1_{731-757}$ (111) mimicking peptides have been separately shown to bind to a conserved PB1 binding site on PA subunit, and substantially reduced viral replication of a panel of viruses, including H1N1 and H5N1.

Advances in structural interactions between human broadly neutralizing antibodies and HA have directed several synthetic proteins and peptides that inhibit fusogenic conformational changes in the endosome. A panel of synthetic HA binder (HB) scaffold proteins have been shown to bind with the conserved region of the stems of HA protein $(112,113)$. HB36 and HB80 were shown to bind $\mathrm{H} 1$ and $\mathrm{H} 5 \mathrm{HA}$ stem with nanomolar affinity, and inhibited low-pH-induced conformational changes. HB80 also displayed similar levels of neutralization compared with the broadly neutralizing antibodies. Furthermore, Kadam et al. recently constructed a series of small cyclic peptides that resemble the epitopes of the stem-targeting broadly neutralizing antibodies (114). These peptidic fusion inhibitors bound to the conserved HA stem epitope at nanomolar affinity, and inhibited HA conformational change at low $\mathrm{pH}$. Similar design strategies could also be applied to other conserved sites on HA, or to other virulence factors of IAV.

As PB1-F2 and NS1 are two major virulence factors that promote pro-inflammatory cytokine storm and inhibit important antiviral immunity, it is interesting to note that there has been no progress in identifying peptides that specifically target these two factors. Ideal therapeutic peptides need to either shield hosts from IAV internalization, or have anti-inflammatory properties without affecting or even increasing antiviral immune responses to infection. These peptides, therefore, are an attractive direction for therapeutics against IAVs, and may be combined with current antiviral drugs to increase the effectiveness of the anti-influenza treatment. The efficacy of peptide-based drugs as preventive agents, however, needs to be further explored, particularly in transmission models such as ferrets.

\section{CONCLUSION}

The constant genetic mutations, recombination, and selection pressure ensure the survival of IAV and its continued threat to mankind. This rapid and threatening evolution of IAV enables the virus to infect multiple species, and with its superior immunomodulatory tactics, the emergence of novel IAVs is unpredictable and inevitable.

HA is the key for the virus to infect, spread, and to create a pandemic. Understanding the absolute molecular requirements for IAV to cross species barriers and achieve sustainable human transmission, and/or aerosol-airborne transmissibility switch will be vital in the development of next-generation virus-targeted therapeutics. This is crucial as the potential scenario of future IAV pandemics is likely to start with a lack of an IAV-specific vaccine, and current antiviral drugs would be available, but with questionable beneficial 
effect. The efficacy of peptide-based antiviral drugs as preventative agents will be an important first line of defense. However, when infection occurs, inhibiting viral polymerases with specific anti$\mathrm{PB} 1 / 2 / \mathrm{PA}$ peptides may also be feasible.

The dynamic duet of PB1-F2 and NS1 effectively controls the host cellular machineries for efficient viral replication, while instigating severe cytokine storm and disarming the host innate antiviral immunity in the infected individuals. Neutralization of their functions would be desirable to reduce infection and potentially fatal symptoms. By the time the infected individuals are admitted to hospital with symptoms, the virus would have shed. Future therapeutics will, therefore, also need to be host-targeted to treat symptoms such as the exaggerated airway or systemic inflammation that is left behind by the virus.

\section{REFERENCES}

1. Hurt AC, Hardie K, Wilson NJ, Deng YM, Osbourn M, Leang SK, et al. Characteristics of a widespread community cluster of H275Y oseltamivir-resistant $\mathrm{A}(\mathrm{H} 1 \mathrm{N1}) \mathrm{pdm} 09$ influenza in Australia. J Infect Dis (2012) 206(2):148-57. doi:10.1093/infdis/jis337

2. Hurt AC, Hardie K, Wilson NJ, Deng YM, Osbourn M, Gehrig N, et al. Community transmission of oseltamivir-resistant A(H1N1)pdm09 influenza. N Engl J Med (2011) 365(26):2541-2. doi:10.1056/NEJMc1111078

3. Lamb RA, Choppin PW. The gene structure and replication of influenza virus. Annu Rev Biochem (1983) 52:467-506. doi:10.1146/annurev. bi.52.070183.002343

4. Palese P, Shaw ML, editors. Orthomyxoviridae: The Viruses and Their Replication. Philadelphia: Lippincott Williams \& Wilkins (2007).

5. Zebedee SL, Lamb RA. Influenza A virus M2 protein: monoclonal antibody restriction of virus growth and detection of M2 in virions. J Virol (1988) 62(8):2762-72.

6. Baudin F, Bach C, Cusack S, Ruigrok RW. Structure of influenza virus RNP. I. Influenza virus nucleoprotein melts secondary structure in panhandle RNA and exposes the bases to the solvent. EMBO J (1994) 13(13):3158-65.

7. Compans RW, Content J, Duesberg PH. Structure of the ribonucleoprotein of influenza virus. J Virol (1972) 10(4):795-800.

8. Murti KG, Webster RG, Jones IM. Localization of RNA polymerases on influenza viral ribonucleoproteins by immunogold labeling. Virology (1988) 164(2):562-6. doi:10.1016/0042-6822(88)90574-0

9. Carr CM, Kim PS. Flu virus invasion: halfway there. Science (1994) 266(5183):234-6. doi:10.1126/science.7939658

10. Hernandez LD, Hoffman LR, Wolfsberg TG, White JM. Virus-cell and cellcell fusion. Annu Rev Cell Dev Biol (1996) 12:627-61. doi:10.1146/annurev. cellbio.12.1.627

11. Whittaker GR, Helenius A. Nuclear import and export of viruses and virus genomes. Virology (1998) 246(1):1-23. doi:10.1006/viro.1998.9165

12. Zhirnov OP. Isolation of matrix protein M1 from influenza viruses by acid-dependent extraction with nonionic detergent. Virology (1992) 186(1):324-30. doi:10.1016/0042-6822(92)90090-C

13. Bui M, Whittaker G, Helenius A. Effect of M1 protein and low $\mathrm{pH}$ on nuclear transport of influenza virus ribonucleoproteins. J Virol (1996) 70(12):8391-401.

14. Martin K, Helenius A. Transport of incoming influenza virus nucleocapsids into the nucleus. J Virol (1991) 65(1):232-44.

15. Scheiffele $P$, Roth MG, Simons K. Interaction of influenza virus haemagglutinin with sphingolipid-cholesterol membrane domains via its transmembrane domain. EMBO J (1997) 16(18):5501-8. doi:10.1093/emboj/16.18.5501

16. Zhang J, Pekosz A, Lamb RA. Influenza virus assembly and lipid raft microdomains: a role for the cytoplasmic tails of the spike glycoproteins. J Virol (2000) 74(10):4634-44. doi:10.1128/JVI.74.10.4634-4644.2000

17. Ito T, Suzuki Y, Mitnaul L, Vines A, Kida H, Kawaoka Y. Receptor specificity of influenza A viruses correlates with the agglutination of erythrocytes from different animal species. Virology (1997) 227(2):493-9. doi:10.1006/ viro. 1996.8323
Peptide-based therapeutics may be the new generation of antiviral drugs, and would be protective against multiple strains/subtypes of IAV if targeted at the essential and conserved regions. This together with ever-improving nanoparticle delivery technologies could be the future of drug design and intracellular delivery system, and must be explored in the calm before the storm.

The great 1918 influenza pandemic happened before, and it will happen again.

\section{AUTHOR CONTRIBUTIONS}

AH conceptualized and wrote the manuscript.

18. Ito T, Suzuki Y, Suzuki T, Takada A, Horimoto T, Wells K, et al. Recognition of N-glycolylneuraminic acid linked to galactose by the alpha2,3 linkage is associated with intestinal replication of influenza A virus in ducks. J Virol (2000) 74(19):9300-5. doi:10.1128/JVI.74.19.9300-9305.2000

19. Ryan-Poirier K, Suzuki Y, Bean WJ, Kobasa D, Takada A, Ito T, et al. Changes in $\mathrm{H} 3$ influenza A virus receptor specificity during replication in humans. Virus Res (1998) 56(2):169-76. doi:10.1016/ S0168-1702(98)00067-7

20. Rogers GN, Paulson JC. Receptor determinants of human and animal influenza virus isolates: differences in receptor specificity of the $\mathrm{H} 3$ hemagglutinin based on species of origin. Virology (1983) 127(2):361-73. doi:10.1016/0042-6822(83)90150-2

21. Baum LG, Paulson JC. Sialyloligosaccharides of the respiratory epithelium in the selection of human influenza virus receptor specificity. Acta Histochem (1990) 40:35-8.

22. SuzukiY,Matsunaga M,MatsumotoM.N-Acetylneuraminyllactosylceramide, GM3-NeuAc, a new influenza A virus receptor which mediates the adsorption-fusion process of viral infection. Binding specificity of influenza virus A/ Aichi/2/68 (H3N2) to membrane-associated GM3 with different molecular species of sialic acid. J Biol Chem (1985) 260(3):1362-5.

23. Ito T, Suzuki Y, Takada A, Kawamoto A, Otsuki K, Masuda H, et al. Differences in sialic acid-galactose linkages in the chicken egg amnion and allantois influence human influenza virus receptor specificity and variant selection. J Virol (1997) 71(4):3357-62.

24. Ito T, Couceiro JN, Kelm S, Baum LG, Krauss S, Castrucci MR, et al. Molecular basis for the generation in pigs of influenza A viruses with pandemic potential. J Virol (1998) 72(9):7367-73.

25. Shinde V, Bridges CB, Uyeki TM, Shu B, Balish A, Xu X, et al. Triple-reassortant swine influenza A (H1) in humans in the United States, 2005-2009. N Engl J Med (2009) 360(25):2616-25. doi:10.1056/NEJMoa0903812

26. Trifonov V, Khiabanian H, Rabadan R. Geographic dependence, surveillance, and origins of the 2009 influenza A (H1N1) virus. N Engl J Med (2009) 361(2):115-9. doi:10.1056/NEJMp0904572

27. Connor RJ, Kawaoka Y, Webster RG, Paulson JC. Receptor specificity in human, avian, and equine $\mathrm{H} 2$ and $\mathrm{H} 3$ influenza virus isolates. Virology (1994) 205(1):17-23. doi:10.1006/viro.1994.1615

28. Rogers GN, D'Souza BL. Receptor binding properties of human and animal H1 influenza virus isolates. Virology (1989) 173(1):317-22. doi:10.1016/0042-6822(89)90249-3

29. Imai M, Watanabe T, Hatta M, Das SC, Ozawa M, Shinya K, et al. Experimental adaptation of an influenza $\mathrm{H} 5 \mathrm{HA}$ confers respiratory droplet transmission to a reassortant H5 HA/H1N1 virus in ferrets. Nature (2012) 486(7403):420-8. doi:10.1038/nature 10831

30. Herfst S, Schrauwen EJ, Linster M, Chutinimitkul S, de Wit E, Munster VJ, et al. Airborne transmission of influenza A/H5N1 virus between ferrets. Science (2012) 336(6088):1534-41. doi:10.1126/science.1213362

31. Biasini M, Bienert S, Waterhouse A, Arnold K, Studer G, Schmidt T, et al. SWISS-MODEL: modelling protein tertiary and quaternary structure using evolutionary information. Nucleic Acids Res (2014) 42(Web Server issue):W252-8. doi:10.1093/nar/gku340 
32. Russier M, Yang G, Rehg JE, Wong SS, Mostafa HH, Fabrizio TP, et al. Molecular requirements for a pandemic influenza virus: an acid-stable hemagglutinin protein. Proc Natl Acad Sci U S A (2016) 113(6):1636-41. doi:10.1073/pnas.1524384113

33. Cummings RD, Soderquist AM, Carpenter G. The oligosaccharide moieties of the epidermal growth factor receptor in A-431 cells. Presence of complex-type $\mathrm{N}$-linked chains that contain terminal $\mathrm{N}$-acetylgalactosamine residues. J Biol Chem (1985) 260(22):11944-52.

34. Yen HY, Liu YC, Chen NY, Tsai CF, Wang YT, Chen YJ, et al. Effect of sialylation on EGFR phosphorylation and resistance to tyrosine kinase inhibition. Proc Natl Acad Sci U S A (2015) 112(22):6955-60. doi:10.1073/ pnas. 1507329112

35. Hsu AC, Starkey MR, Hanish I, Parsons K, Haw TJ, Howland LJ, et al. Targeting PI3K-p110alpha suppresses influenza virus infection in chronic obstructive pulmonary disease. Am J Respir Crit Care Med (2015) 191(9):1012-23. doi:10.1164/rccm.201501-0188OC

36. Kedzierski L, Tate MD, Hsu AC, Kolesnik TB, Linossi EM, Dagley L, et al. Suppressor of cytokine signaling (SOCS) 5 ameliorates influenza infection via inhibition of EGFR signaling. Elife (2017) 6:e20444. doi:10.7554/eLife.20444

37. Eierhoff T, Hrincius ER, Rescher U, Ludwig S, Ehrhardt C. The epidermal growth factor receptor (EGFR) promotes uptake of influenza A viruses (IAV) into host cells. PLoS Pathog (2010) 6(9):e1001099. doi:10.1371/journal. ppat.1001099

38. Glaser L, Conenello G, Paulson J, Palese P. Effective replication of human influenza viruses in mice lacking a major alpha2,6 sialyltransferase. Virus Res (2007) 126(1-2):9-18. doi:10.1016/j.virusres.2007.01.011

39. Hsu AC, Barr I, Hansbro PM, Wark PA. Human influenza is more effective than avian influenza at antiviral suppression in airway cells. Am J Respir Cell Mol Biol (2011) 44(6):906-13. doi:10.1165/rcmb.2010-0157OC

40. Nunes-Correia I, Eulalio A, Nir S, Pedroso de Lima MC. Caveolae as an additional route for influenza virus endocytosis in MDCK cells. Cell $\mathrm{Mol}$ Biol Lett (2004) 9(1):47-60.

41. Rapoport EM, Mochalova LV, Gabius HJ, Romanova J, Bovin NV. Search for additional influenza virus to cell interactions. Glycoconj J (2006) 23(1-2):115-25. doi:10.1007/s10719-006-5444-x

42. Clements ML, Subbarao EK, Fries LF, Karron RA, London WT, Murphy BR. Use of single-gene reassortant viruses to study the role of avian influenza A virus genes in attenuation of wild-type human influenza A virus for squirrel monkeys and adult human volunteers. J Clin Microbiol (1992) 30(3):655-62.

43. Subbarao EK, London W, Murphy BR. A single amino acid in the PB2 gene of influenza A virus is a determinant of host range. J Virol (1993) 67(4):1761-4.

44. de Wit E, Munster VJ, van Riel D, Beyer WE, Rimmelzwaan GF, Kuiken $\mathrm{T}$, et al. Molecular determinants of adaptation of highly pathogenic avian influenza H7N7 viruses to efficient replication in the human host. J Virol (2010) 84(3):1597-606. doi:10.1128/JVI.01783-09

45. Li Z, Chen H, Jiao P, Deng G, Tian G, Li Y, et al. Molecular basis of replication of duck H5N1 influenza viruses in a mammalian mouse model. J Virol (2005) 79(18):12058-64. doi:10.1128/JVI.79.18.12058-12064.2005

46. Gabriel G, Dauber B, WolffT, Planz O, Klenk HD, Stech J. The viral polymerase mediates adaptation of an avian influenza virus to a mammalian host. Proc Natl Acad Sci U S A (2005) 102(51):18590-5. doi:10.1073/pnas.0507415102

47. Le QM, Sakai-Tagawa Y, Ozawa M, Ito M, Kawaoka Y. Selection of H5N1 influenza virus PB2 during replication in humans. J Virol (2009) 83(10):5278-81. doi:10.1128/JVI.00063-09

48. Kageyama T, Fujisaki S, Takashita E, Xu H, Yamada S, Uchida Y, et al. Genetic analysis of novel avian A(H7N9) influenza viruses isolated from patients in China, February to April 2013. Euro Surveill (2013) 18(15):20453

49. Gao R, Cao B, Hu Y, Feng Z, Wang D, Hu W, et al. Human infection with a novel avian-origin influenza A (H7N9) virus. N Engl J Med (2013) 368(20):1888-97. doi:10.1056/NEJMoa1304459

50. Kawaoka Y, Krauss S, Webster RG. Avian-to-human transmission of the PB1 gene of influenza A viruses in the 1957 and 1968 pandemics. J Virol (1989) 63(11):4603-8.

51. Snyder MH, Buckler-White AJ, London WT, Tierney EL, Murphy BR. The avian influenza virus nucleoprotein gene and a specific constellation of avian and human virus polymerase genes each specify attenuation of avian-human influenza A/Pintail/79 reassortant viruses for monkeys. J Virol (1987) 61(9):2857-63.
52. Chen W, Calvo PA, Malide D, Gibbs J, Schubert U, Bacik I, et al. A novel influenza A virus mitochondrial protein that induces cell death. Nat Med (2001) 7(12):1306-12. doi:10.1038/nm1201-1306

53. Zamarin D, Garcia-Sastre A, Xiao X, Wang R, Palese P. Influenza virus PB1F2 protein induces cell death through mitochondrial ANT3 and VDAC1. PLoS Pathog (2005) 1(1):e4. doi:10.1371/journal.ppat.0010004

54. Yoshizumi T, Ichinohe T, Sasaki O, Otera H, Kawabata S, Mihara K, et al. Influenza A virus protein PB1-F2 translocates into mitochondria via Tom40 channels and impairs innate immunity. Nat Commun (2014) 5:4713. doi: $10.1038 /$ ncomms5713

55. Gack MU, Shin YC, Joo CH, Urano T, Liang C, Sun L, et al. TRIM25 RINGfinger E3 ubiquitin ligase is essential for RIG-I-mediated antiviral activity. Nature (2007) 446(7138):916-20. doi:10.1038/nature05732

56. Seth RB, Sun L, Ea CK, Chen ZJ. Identification and characterization of MAVS, a mitochondrial antiviral signaling protein that activates NF-kappaB and IRF 3. Cell (2005) 122(5):669-82. doi:10.1016/j.cell.2005.08.012

57. Fitzgerald KA, McWhirter SM, Faia KL, Rowe DC, Latz E, Golenbock DT, et al. IKKepsilon and TBK1 are essential components of the IRF3 signaling pathway. Nat Immunol (2003) 4(5):491-6. doi:10.1038/ni921

58. Stark GR, Kerr IM, Williams BR, Silverman RH, Schreiber RD. How cells respond to interferons. Annu Rev Biochem (1998) 67:227-64. doi:10.1146/ annurev.biochem.67.1.227

59. Alexopoulou L, Holt AC, Medzhitov R, Flavell RA. Recognition of double-stranded RNA and activation of NF-kappaB by toll-like receptor 3 . Nature (2001) 413(6857):732-8. doi:10.1038/35099560

60. Chaouat A, Savale L, Chouaid C, Tu L, Sztrymf B, Canuet M, et al. Role for interleukin-6 in COPD-related pulmonary hypertension. Chest (2009) 136(3):678-87. doi:10.1378/chest.08-2420

61. Takeda K, Kaisho T, Akira S. Toll-like receptors. Annu Rev Immunol (2003) 21:335-76. doi:10.1146/annurev.immunol.21.120601.141126

62. Varga ZT, Grant A, Manicassamy B, Palese P. Influenza virus protein PB1-F2 inhibits the induction of type I interferon by binding to MAVS and decreasing mitochondrial membrane potential. J Virol (2012) 86(16):8359-66. doi:10.1128/JVI.01122-12

63. Varga ZT, Ramos I, Hai R, Schmolke M, Garcia-Sastre A, Fernandez-Sesma $A$, et al. The influenza virus protein PB1-F2 inhibits the induction of type I interferon at the level of the MAVS adaptor protein. PLoS Pathog (2011) 7(6):e1002067. doi:10.1371/journal.ppat.1002067

64. McAuley JL, Hornung F, Boyd KL, Smith AM, McKeon R, Bennink J, et al. Expression of the 1918 influenza A virus PB1-F2 enhances the pathogenesis of viral and secondary bacterial pneumonia. Cell Host Microbe (2007) 2(4):240-9. doi:10.1016/j.chom.2007.09.001

65. Conenello GM, Zamarin D, Perrone LA, Tumpey T, Palese P. A single mutation in the PB1-F2 of H5N1 (HK/97) and 1918 influenza A viruses contributes to increased virulence. PLoS Pathog (2007) 3(10):1414-21. doi:10.1371/journal.ppat.0030141

66. McAuley JL, Tate MD, MacKenzie-Kludas CJ, Pinar A, Zeng W, Stutz A, et al. Activation of the NLRP3 inflammasome by IAV virulence protein PB1-F2 contributes to severe pathophysiology and disease. PLoS Pathog (2013) 9(5):e1003392. doi:10.1371/journal.ppat.1003392

67. Zamarin D, Ortigoza MB, Palese P. Influenza A virus PB1-F2 protein contributes to viral pathogenesis in mice. J Virol (2006) 80(16):7976-83. doi:10.1128/JVI.00415-06

68. Pinar A, Dowling JK, Bitto NJ, Robertson AA, Latz E, Stewart CR, et al. $\mathrm{PB} 1-\mathrm{F} 2$ peptide derived from avian influenza A virus H7N9 induces inflammation via activation of the NLRP3 inflammasome. J Biol Chem (2017) 292(3):826-36. doi:10.1074/jbc.M116.756379

69. Chanturiya AN, Basanez G, Schubert U, Henklein P, Yewdell JW, Zimmerberg J. PB1-F2, an influenza A virus-encoded proapoptotic mitochondrial protein, creates variably sized pores in planar lipid membranes. J Virol (2004) 78(12):6304-12. doi:10.1128/ JVI.78.12.6304-6312.2004

70. Bruns K, Studtrucker N, Sharma A, Fossen T, Mitzner D, Eissmann A, et al. Structural characterization and oligomerization of PB1-F2, a proapoptotic influenza A virus protein. J Biol Chem (2007) 282(1):353-63. doi:10.1074/ jbc.M606494200

71. Isaacs A, Lindenmann J, Valentine RC. Virus interference. II. Some properties of interferon. Proc R Soc Lond B Biol Sci (1957) 147(927):268-73. doi:10.1098/rspb.1957.0049 
72. Isaacs A, Lindenmann J. Virus interference. I. The interferon. Proc R Soc Lond B Biol Sci (1957) 147(927):258-67. doi:10.1098/rspb.1957.0048

73. HatadaE, Fukuda R. Binding of influenza A virus NS1 protein to dsRNA in vitro. J Gen Virol (1992) 73(Pt 12):3325-9. doi:10.1099/0022-1317-73-12-3325

74. Privalsky ML, Penhoet EE. The structure and synthesis of influenza virus phosphoproteins. J Biol Chem (1981) 256(11):5368-76.

75. Chien CY, Xu Y, Xiao R, Aramini JM, Sahasrabudhe PV, Krug RM, et al. Biophysical characterization of the complex between double-stranded RNA and the N-terminal domain of the NS1 protein from influenza A virus: evidence for a novel RNA-binding mode. Biochemistry (2004) 43(7):1950-62. doi:10.1021/bi030176o

76. Qian XY, Chien CY, Lu Y, Montelione GT, Krug RM. An amino-terminal polypeptide fragment of the influenza virus NS1 protein possesses specific RNA-binding activity and largely helical backbone structure. RNA (1995) 1(9):948-56.

77. Chen Z, Li Y, Krug RM. Influenza A virus NS1 protein targets poly(A)-binding protein II of the cellular 3'-end processing machinery. EMBO J (1999) 18(8):2273-83. doi:10.1093/emboj/18.8.2273

78. Nemeroff ME, Barabino SM, Li Y, Keller W, Krug RM. Influenza virus NS1 protein interacts with the cellular $30 \mathrm{kDa}$ subunit of CPSF and inhibits 3' end formation of cellular pre-mRNAs. Mol Cell (1998) 1(7):991-1000. doi:10.1016/S1097-2765(00)80099-4

79. Noah DL, Twu KY, Krug RM. Cellular antiviral responses against influenza A virus are countered at the posttranscriptional level by the viral NS1A protein via its binding to a cellular protein required for the 3 ' end processing of cellular pre-mRNAS. Virology (2003) 307(2):386-95. doi:10.1016/ S0042-6822(02)00127-7

80. Marazzi I, Ho JS, Kim J, Manicassamy B, Dewell S, Albrecht RA, et al. Suppression of the antiviral response by an influenza histone mimic. Nature (2012) 483(7390):428-33. doi:10.1038/nature10892

81. Mibayashi M, Martinez-Sobrido L, Loo YM, Cardenas WB, Gale M Jr, Garcia-Sastre A. Inhibition of retinoic acid-inducible gene I-mediated induction of beta interferon by the NS1 protein of influenza A virus. J Virol (2007) 81(2):514-24. doi:10.1128/JVI.01265-06

82. Talon J, Horvath CM, Polley R, Basler CF, Muster T, Palese P, et al. Activation of interferon regulatory factor 3 is inhibited by the influenza A virus NS1 protein. J Virol (2000) 74(17):7989-96. doi:10.1128/JVI.74.17.7989-7996.2000

83. Gack MU, Albrecht RA, Urano T, Inn KS, Huang IC, Carnero E, et al. Influenza A virus NS1 targets the ubiquitin ligase TRIM25 to evade recognition by the host viral RNA sensor RIG-I. Cell Host Microbe (2009) 5(5):439-49. doi:10.1016/j.chom.2009.04.006

84. Bornholdt ZA, Prasad BV. X-ray structure of NS1 from a highly pathogenic H5N1 influenza virus. Nature (2008) 456(7224):985-8. doi:10.1038/ nature 07444

85. Wang X, Basler CF, Williams BR, Silverman RH, Palese P, Garcia-Sastre A. Functional replacement of the carboxy-terminal two-thirds of the influenza A virus NS1 protein with short heterologous dimerization domains. J Virol (2002) 76(24):12951-62. doi:10.1128/JVI.76.24.12951-12962.2002

86. Lu Y, Wambach M, Katze MG, Krug RM. Binding of the influenza virus NS1 protein to double-stranded RNA inhibits the activation of the protein kinase that phosphorylates the elF-2 translation initiation factor. Virology (1995) 214(1):222-8. doi:10.1006/viro.1995.9937

87. Bergmann M, Garcia-Sastre A, Carnero E, Pehamberger H, Wolff K, Palese P, et al. Influenza virus NS1 protein counteracts PKR-mediated inhibition of replication. J Virol (2000) 74(13):6203-6. doi:10.1128/JVI.74.13.6203-6206.2000

88. Dauber B, Schneider J, Wolff T. Double-stranded RNA binding of influenza $B$ virus nonstructural NS1 protein inhibits protein kinase R but is not essential to antagonize production of alpha/beta interferon. J Virol (2006) 80(23):11667-77. doi:10.1128/JVI.01142-06

89. Li S, Min JY, Krug RM, Sen GC. Binding of the influenza A virus NS1 protein to PKR mediates the inhibition of its activation by either PACT or double-stranded RNA. Virology (2006) 349(1):13-21. doi:10.1016/j.virol.2006.01.005

90. Min JY, Li S, Sen GC, Krug RM. A site on the influenza A virus NS1 protein mediates both inhibition of PKR activation and temporal regulation of viral RNA synthesis. Virology (2007) 363(1):236-43. doi:10.1016/j.virol.2007.01.038

91. Min JY, Krug RM. The primary function of RNA binding by the influenza A virus NS1 protein in infected cells: inhibiting the 2'-5' oligo (A) synthetase/RNase L pathway. Proc Natl Acad Sci U S A (2006) 103(18):7100-5. doi:10.1073/pnas.0602184103
92. Hale BG, Jackson D, Chen YH, Lamb RA, Randall RE. Influenza A virus NS1 protein binds p85beta and activates phosphatidylinositol-3-kinase signaling. Proc Natl Acad Sci U S A (2006) 103(38):14194-9. doi:10.1073/ pnas. 0606109103

93. Li Y, Anderson DH, Liu Q, Zhou Y. Mechanism of influenza A virus NS1 protein interaction with the p85beta, but not the p85alpha, subunit of phosphatidylinositol 3-kinase (PI3K) and up-regulation of PI3K activity. J Biol Chem (2008) 283(34):23397-409. doi:10.1074/jbc.M802737200

94. Shin YK, Li Y, Liu Q, Anderson DH, Babiuk LA, Zhou Y. SH3 binding motif 1 in influenza A virus NS1 protein is essential for PI3K/Akt signaling pathway activation. J Virol (2007) 81(23):12730-9. doi:10.1128/JVI.01427-07

95. Kochs G, Koerner I, Thiel L, Kothlow S, Kaspers B, Ruggli N, et al. Properties of H7N7 influenza A virus strain SC35M lacking interferon antagonist NS1 in mice and chickens. JGen Virol (2007) 88(Pt 5):1403-9. doi:10.1099/ vir.0.82764-0

96. Cauthen AN, Swayne DE, Sekellick MJ, Marcus PI, Suarez DL. Amelioration of influenza virus pathogenesis in chickens attributed to the enhanced interferon-inducing capacity of a virus with a truncated NS1 gene. J Virol (2007) 81(4):1838-47. doi:10.1128/JVI.01667-06

97. Solorzano A, Webby RJ, Lager KM, Janke BH, Garcia-Sastre A, Richt JA. Mutations in the NS1 protein of swine influenza virus impair anti-interferon activity and confer attenuation in pigs. J Virol (2005) 79(12):7535-43. doi:10.1128/JVI.79.12.7535-7543.2005

98. Zhu Q, Yang H, Chen W, Cao W, Zhong G, Jiao P, et al. A naturally occurring deletion in its NS gene contributes to the attenuation of an $\mathrm{H} 5 \mathrm{~N} 1$ swine influenza virus in chickens. J Virol (2008) 82(1):220-8. doi:10.1128/JVI.00978-07

99. Twu KY, Kuo RL, Marklund J, Krug RM. The H5N1 influenza virus NS genes selected after 1998 enhance virus replication in mammalian cells. J Virol (2007) 81(15):8112-21. doi:10.1128/JVI.00006-07

100. Hsu AC, Parsons K, Barr I, Lowther S, Middleton D, Hansbro PM, et al. Critical role of constitutive type I interferon response in bronchial epithelial cell to influenza infection. PLoS One (2012) 7(3):e32947. doi:10.1371/ journal.pone.0032947

101. Chambers BS, Parkhouse K, Ross TM, Alby K, Hensley SE. Identification of hemagglutinin residues responsible for $\mathrm{H} 3 \mathrm{~N} 2$ antigenic drift during the 2014-2015 influenza season. Cell Rep (2015) 12(1):1-6. doi:10.1016/j. celrep.2015.06.005

102. Nguyen-Van-Tam JS, Venkatesan S, Muthuri SG, Myles PR. Neuraminidase inhibitors: who, when, where? Clin Microbiol Infect (2015) 21(3):222-5. doi:10.1016/j.cmi.2014.11.020

103. Wolkewitz M, Schumacher M. Neuraminidase inhibitors and hospital mortality in British patients with H1N1 influenza A: a re-analysis of observational data. PLoS One (2016) 11(9):e0160430. doi:10.1371/journal.pone. 0160430

104. Chen Q, Guo Y. Influenza viral hemagglutinin peptide inhibits influenza viral entry by shielding the host receptor. ACS Infect Dis (2016) 2(3):187-93. doi:10.1021/acsinfecdis.5b00139

105. Jones JC, Turpin EA, Bultmann H, Brandt CR, Schultz-Cherry S. Inhibition of influenza virus infection by a novel antiviral peptide that targets viral attachment to cells. J Virol (2006) 80(24):11960-7. doi:10.1128/JVI.01678-06

106. Nicol MQ, Ligertwood Y, Bacon MN, Dutia BM, Nash AA. A novel family of peptides with potent activity against influenza A viruses. J Gen Virol (2012) 93(Pt 5):980-6. doi:10.1099/vir.0.038679-0

107. Cederlund A, Gudmundsson GH, Agerberth B. Antimicrobial peptides important in innate immunity. FEBS $J$ (2011) 278(20):3942-51. doi:10.1111/j.1742-4658.2011.08302.x

108. Gutsmann T, Razquin-Olazaran I, Kowalski I, Kaconis Y, Howe J, Bartels R, et al. New antiseptic peptides to protect against endotoxin-mediated shock. Antimicrob Agents Chemother (2010) 54(9):3817-24. doi:10.1128/AAC.00534-10

109. Hoffmann J, Schneider C, Heinbockel L, Brandenburg K, Reimer R, Gabriel G. A new class of synthetic anti-lipopolysaccharide peptides inhibits influenza A virus replication by blocking cellular attachment. Antiviral Res (2014) 104:23-33. doi:10.1016/j.antiviral.2014.01.015

110. Ghanem A, Mayer D, Chase G, Tegge W, Frank R, Kochs G, et al. Peptidemediated interference with influenza A virus polymerase. J Virol (2007) 81(14):7801-4. doi:10.1128/JVI.00724-07

111. Li C, Ba Q, Wu A, Zhang H, Deng T, Jiang T. A peptide derived from the C-terminus of PB1 inhibits influenza virus replication by interfering with viral polymerase assembly. FEBS J (2013) 280(4):1139-49. doi:10.1111/febs.12107 
112. Fleishman SJ, Whitehead TA, Ekiert DC, Dreyfus C, Corn JE, Strauch EM, et al. Computational design of proteins targeting the conserved stem region of influenza hemagglutinin. Science (2011) 332(6031):816-21. doi:10.1126/science.1202617

113. Whitehead TA, Chevalier A, Song Y, Dreyfus C, Fleishman SJ, De Mattos $\mathrm{C}$, et al. Optimization of affinity, specificity and function of designed influenza inhibitors using deep sequencing. Nat Biotechnol (2012) 30(6):543-8. doi:10.1038/nbt.2214

114. Kadam RU, Juraszek J, Brandenburg B, Buyck C, Schepens WBG, Kesteleyn B, et al. Potent peptidic fusion inhibitors of influenza virus. Science (2017) 358(6362):496-502. doi:10.1126/science.aan 0516
Conflict of Interest Statement: The author declares that the research was conducted in the absence of any commercial or financial relationships that could be construed as a potential conflict of interest.

Copyright $\odot 2018 \mathrm{Hsu}$. This is an open-access article distributed under the terms of the Creative Commons Attribution License (CC BY). The use, distribution or reproduction in other forums is permitted, provided the original author(s) and the copyright owner are credited and that the original publication in this journal is cited, in accordance with accepted academic practice. No use, distribution or reproduction is permitted which does not comply with these terms. 